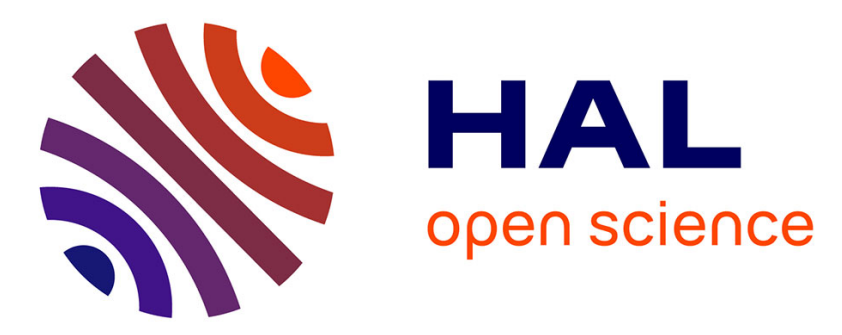

\title{
Transient charge density wave dynamics in pure, non-stoichiometric and V-doped blue bronzes A0.30MoO3 $(\mathrm{A}=\mathrm{K}, \mathrm{Rb})$
}

J. Dumas, A. Arbaoui

\section{- To cite this version:}

J. Dumas, A. Arbaoui. Transient charge density wave dynamics in pure, non-stoichiometric and Vdoped blue bronzes A0.30MoO3 ( $\mathrm{A}=\mathrm{K}, \mathrm{Rb})$. Journal de Physique IV Proceedings, 1993, 03 (C2), pp.C2-179-C2-184. 10.1051/jp4:1993236 . jpa-00251319

\section{HAL Id: jpa-00251319 https://hal.science/jpa-00251319}

Submitted on 1 Jan 1993

HAL is a multi-disciplinary open access archive for the deposit and dissemination of scientific research documents, whether they are published or not. The documents may come from teaching and research institutions in France or abroad, or from public or private research centers.
L'archive ouverte pluridisciplinaire HAL, est destinée au dépôt et à la diffusion de documents scientifiques de niveau recherche, publiés ou non, émanant des établissements d'enseignement et de recherche français ou étrangers, des laboratoires publics ou privés. 


\title{
Transient charge density wave dynamics in pure, non-stoichiometric and V-doped blue bronzes $\mathrm{A}_{0.30} \mathrm{MoO}_{3}(\mathrm{~A}=\mathrm{K}, \mathrm{Rb})$
}

\author{
J. DUMAS and A. ARBAOUI \\ CNRS, Laboratoire d'Etudes des Propriétés Electroniques des Solides, Associé à l'Université J. Fourier, \\ BP. 166, 38042 Grenoble cedex 9, France
}

\begin{abstract}
We report the observation of the transient voltage response to current pulses in pure, non-stoichiometric and $\mathrm{V}$-doped blue bronzes $\mathrm{A}_{0.30} \mathrm{MoO}_{3}(\mathrm{~A}=\mathrm{K}, \mathrm{Rb})$ near threshold for depinning of the charge density wave. The transient response waveforms involve overshoot and sluggish behaviors at the leading edge of the pulse in pure samples, weak sluggish response in non-stoichiometric samples and strong sluggish behavior in V-doped blue bronzes. The time decays of the transient responses obey stretched exponential laws. The characteristic time for the overshoot obeys a power law as a function of the electric field and is divergent as threshold is approached from above, suggesting that depinning can be viewed as a dynamical critical phenomenon. The transient response has also been studied under inhomogeneous conditions when a current pulse is injected in a segment of the sample while a dc current is flowing in a neighboring segment. The different regimes found in the transient response are analyzed in analogy with strain transient and plastic yielding of a crystal and in terms of combined motion of edge and screw charge density wave phase dislocations interacting with impurities.
\end{abstract}

\section{Introduction}

Charge density wave (CDW) dynamics in quasi-one dimensional conductors has been the subject of intensive studies over the past 15 years (1). Below a small finite threshold field $E_{t}$, the CDW is pinned to the underlying lattice because of its interactions with randomly distributed impurities. Above $E_{t}$, remarkable non-linear conductivity due to the motion of the CDW with respect to the lattice is observed. Because of randomness, a large number of metastable states are present for the CDW involving a broad range of time scales. In the Fukuyama-Lee-Rice model $(2,3)$, the pinning results from the elastic energy of the CDW and the prefered phase at impurity sites. More recently, the CDW depinning as been viewed as a dynamical critical phenomenon (4). Other models involve CDW structural defects such as phase dislocations (5). The analogy between depinning and onset of plastic flow of dislocations in a crystal has been reported (6).

An interesting method of probing the depinning is through the investigation of transient voltage response to current pulses. We have compared the transient voltage response near threshold to current pulses in pure $\mathrm{A}_{0.30} \mathrm{MoO}_{3}(\mathrm{~A}=\mathrm{K}, \mathrm{Rb})$, non-stoichiometric $\mathrm{Rb}_{0.28} \mathrm{MoO}_{3}$ and $\mathrm{V}$-doped $\mathrm{Rb}_{0.30} \mathrm{MoO}_{3}$. The non-linearity above $40 \mathrm{~K}$ arises from the motion of a deformable CDW strongly damped by normal carriers and the abrupt depinning at lower temperatures is ascribed to an increased rigidity of the CDW when the compound becomes an insulator (7). We have previously reported a study of the transient response to current pulses in switching samples (8). The response to current pulses related to pulse sign memory (9) or to pulse duration memory (10) has been reported. The response to single (11) or double pulse (12) has also been investigated. In this work, we report measurements of the transient response to unipolar rectangular current pulses at temperatures above $40 \mathrm{~K}$ for non-switching samples of pure, non-stoichiometric and V-doped blue bronzes. We also report the results of a study of the transient response in a segment of a sample when a dc current is flowing in a neighboring segment. Preliminary results are given in ref. 13. V impurities enter 
substitutionaly on the Mo CDW chains and lead to a broadening of the Peierls transition (14) while in nonstoichiometric samples alcaline vacancies are adjacent to the chains and have no effect on the Peierls transition temperature. We suggest that CDW structural defects, possibly edge and screw CDW phase dislocations, interacting with impurities are involved in these transient responses. An analogy with time dependent plastic behavior in metals submitted to cyclic loading is proposed.

\section{Experimental results}

2a. Transient response

The single crystals were prepared by standard electrolytic reduction of a $\mathrm{A}_{2} \mathrm{MoO}_{4}-\mathrm{MoO}_{3}$ melt. From a comparative microprobe analysis of $\mathrm{Rb}$ blue bronzes also prepared electrolytically according to ref. (15) and provided to us by $P$. Strobel, and of our $R b_{0.30} \mathrm{MoO}_{3}$ samples, a chemical composition $\mathrm{R} b_{0.28} \mathrm{MoO}_{3}$ was found for the former assuming that all the alcaline sites were occupied in the latter. Electrical contacts were obtained by copper electroplating on freshly cleaved samples then silver paste soldering. The transient response of more than forty samples was investigated using the four probe geometry. The computer controlled arrangement consists of a HP 214B pulse generator, a TK2230 storage oscilloscope and TK AM502 differential amplifiers. Typical pulse length was 1ms. A constant current was applied to the sample using a large resistance in series with the pulse generator.

For pure $\mathrm{A}_{0.30} \mathrm{MoO}_{3}$ we have observed three kinds of response with no correlation with the pulse threshold field values $E_{t, p}$ : In Figure 1a, we compare the responses to a sequence of three pulses above $\mathrm{E}_{\mathrm{t}, \mathrm{p}}$ for the three kinds of samples. In sample 1 , the response at the leading edge is always marked by an overshoot; in sample 2 , the response to the first pulse is marked by an overshoot while for the subsequent two pulses a mixed response with overshoot and sluggish behavior is found; in sample 3, the response to the first pulse is marked by a weak overshoot and the subsequent pulses show a sluggish response.

In Figure $1 \mathrm{~b}$, we show the typical voltage response in the case of $\mathrm{Rb}_{0.28} \mathrm{MoO}_{3}$ : the response to the first pulse is extremely sluggish; the subsequent pulses show a sluggish behavior. After a few minutes, in a new sequence of three pulses, the first one shows a weak overshoot at the leading edge. The amplitude of the overshoot in $\mathrm{Rb}_{0.28} \mathrm{MoO}_{3}$ is always smaller than that found in pure blue bronzes.

In V-doped samples, a pronounced sluggish response, illustrated in Figure 2, is always observed.

The pulse threshold field $E_{t, p}$ increases when the pulse width decreases. $E_{t, p}$ is more sensitive to the pulse width in $\mathrm{Rb}_{0.28} \mathrm{MoO}_{3}$ than in V-doped samples, as shown in Figure 3.

The overshoot $\Delta V_{\text {on }}$ is observed at a threshold field $E_{c}$ slightly below the threshold $E_{t, p}$ for depinning obtained on the V-I curves from the measurements of the steady voltage $V$ at the end of the pulse; typically, $\mathrm{E}_{\mathrm{c}}=0.8 \mathrm{E}_{\mathrm{t}, \mathrm{p}}$ at $77 \mathrm{~K} . \Delta \mathrm{V}_{\mathrm{on}} / \mathrm{V}$ increases with applied current pulse amplitude and decreases with temperature. The relaxation $\Delta V_{\text {off }}$ at the trailing edge of the pulse is observed not only near and above $E_{c}$ but also below $\mathrm{E}_{\mathrm{c}}$.

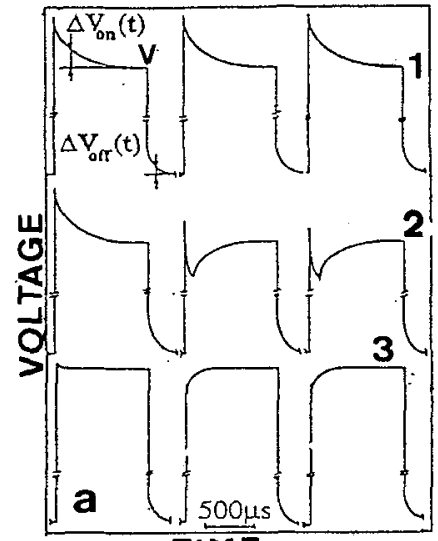

TIME

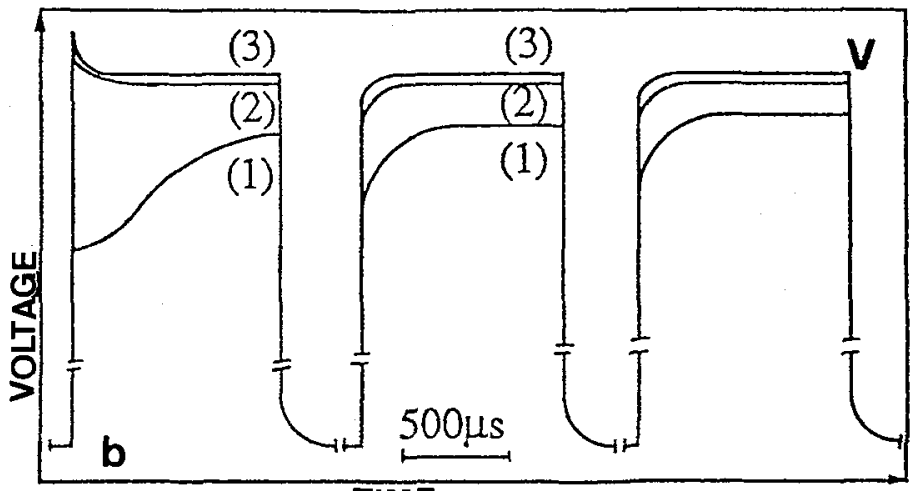

TIME

Figure 1.(a) Voltage response waveform for $\mathrm{K}_{0.30} \mathrm{MoO}_{3}$ samples at $77 \mathrm{~K}$ to a series of three pulses: (1) sample 1 showing overshoot; (2) sample 2 showing overshoot for the first pulse then mixed overshoot and sluggish response for the subsequent pulses; (3) sample 3 showing weak overshoot on the first pulse then sluggish response for the subsequent pulses. Pulse width $t_{w}=1 \mathrm{~ms} ;$ repetition rate $T_{\text {rep }}=5 \mathrm{~ms}$.

(b) Voltage response waveform for a $\mathrm{Rb}_{0.28} \mathrm{MoO}_{3}$ sample at $77 \mathrm{~K} . \mathrm{t}_{\mathrm{w}}=1 \mathrm{~ms} ; \mathrm{T}_{\text {rep }}=5 \mathrm{~ms}$. (1): virgin state;

(2) after 2 minutes; (3) after 4 minutes. 


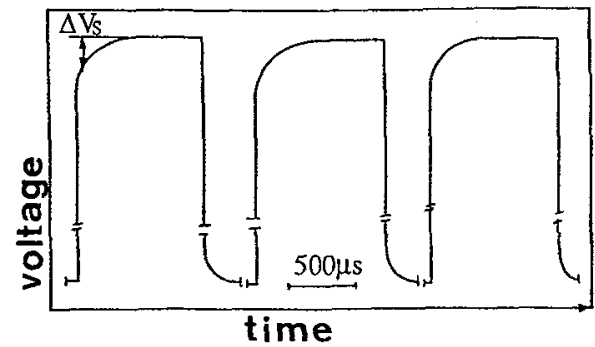

Figure 2. Voltage response waveform for a $\mathrm{Rb}_{0.30} \mathrm{Mo}_{1-\mathrm{x}} \mathrm{V}_{\mathrm{x}} \mathrm{O}_{3}$ sample ; $\mathrm{x}=0.4$ at\%. $\mathrm{T}=77 \mathrm{~K}$. $\mathrm{t}_{\mathrm{w}}=1 \mathrm{~ms}$; $\mathrm{T}_{\text {rep }}=5 \mathrm{~ms}$.

The overshoot amplitude $\Delta \mathrm{V}_{\text {on }}$ and the sluggish response $\Delta \mathrm{V}_{\mathrm{s}}$ are found to obey a stretched exponential time dependence: $\Delta V_{\text {on }} \alpha \exp -\left(t / \tau_{\text {on }}\right)^{\beta}, \Delta V_{s} \alpha \exp -\left(t / \tau_{s}\right) \beta^{\prime}\left(0<\beta, \beta^{\prime}<1\right) . \tau_{\text {on }}$ and $\tau_{s}$ depend on the electric field and on the temperature. The response at the trailing edge $\Delta V_{\text {off }}$ is found to obey also a stretched exponential law. $\tau_{\text {on }}$ and $\tau_{\mathrm{s}}$ are divergent as threshold is approached from above. $\tau_{\text {on }}$ is satisfactorily described using a power law : $\tau_{\text {on }} \alpha\left(\mathrm{E}_{/} \mathrm{E}_{\mathrm{c}}-1\right)^{-\gamma}$ as shown in Figure 4. $\tau_{\mathrm{s}}$ also obeys a power law with an exponent $\gamma=2.5$ in $\mathrm{K}_{0.30} \mathrm{MoO}_{3}, 1.3$ in theV-doped sample and 1.12 in $\mathrm{Rb}_{0.28} \mathrm{MoO}_{3}$ at $77 \mathrm{~K}$. $\tau_{\text {off }}$ increases with the electric field . $\tau_{\text {off }}$ is much larger (resp. much smaller) in $\mathrm{Rb}_{0.28} \mathrm{MoO}_{3}$ (resp. V-doped $\mathrm{Rb}_{0.30} \mathrm{MoO}_{3}$ ) than in pure $\mathrm{A}_{0.30} \mathrm{MoO}_{3}$. These results show that the transient response is correlated with the $\mathrm{CDW}$ impurity pinning strength.
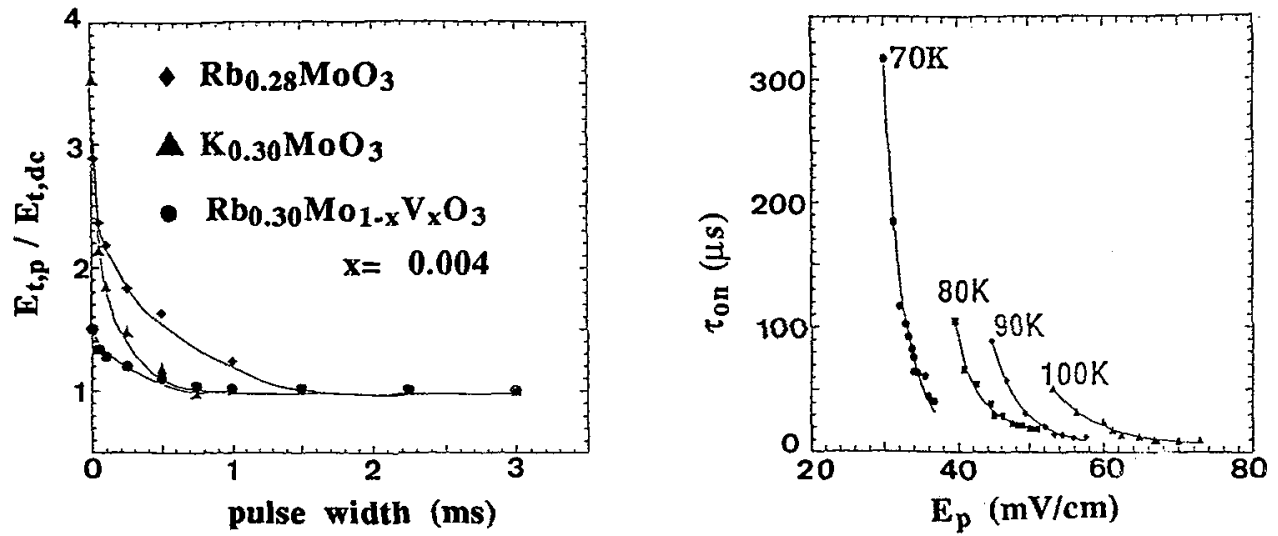

Figure 3. Pulse threshold field normalized to the dc threshold field as a function of the pulse width for pure $\mathrm{K}_{0.30} \mathrm{MoO}_{3}, \mathrm{Rb}_{0.28} \mathrm{MoO}_{3}, \mathrm{Rb}_{0.30} \mathrm{Mo}_{1-\mathrm{x}} \mathrm{V}_{\mathrm{x}} \mathrm{O}_{3} ; \mathrm{x}=0.4 \mathrm{at} \%$. $\mathrm{T}=77 \mathrm{~K}$.

Figure 4. Characteristic time $\tau_{\text {on }}$ as a function of pulse field amplitude at different temperatures for $\mathrm{K}_{0.30} \mathrm{MoO}_{3}$ (sample 1 in Fig.1). Solid line is a fit according to the law: $\tau_{\mathrm{on}} \alpha\left(\mathrm{E} / \mathrm{E}_{\mathrm{c}}-1\right)^{-\gamma} ; \gamma=2$ at $70 \mathrm{~K}$.

2b. Breaking of coherence

Little is known about various aspects concerning the non-local CDW dynamics, related to the finite correlation length of the CDW. Evidence for non-local behavior has been given by several experiments: application of a thermal gradient (5), size effect on the threshold field(16), influence of a current flowing through one part of the sample while voltage response is investigated in another part $(17,18)$. Up to now, depinning under inhomogeneous conditions in the blue bronze $\mathrm{K}_{0.30} \mathrm{MoO}_{3}$ was studied by considering the effect of a thermal gradient along the sample on the narrow band noise (19). Other recent observations of non-local properties of a moving CDW have been given by Csiba et al.(20) who observed propagation of voltage spikes near threshold in a blue bronze sample.

In order to study non-local effects, we have investigated the influence of a dc current flowing through one segment of the sample on the voltage response to unipolar current pulses in a neighboring segment, in pure $\mathrm{K}_{0.30} \mathrm{MoO}_{3}$. 
In Figure 5(a), we show the change of the transient voltage response waveform, measured between probes $(3,4)$ at a fixed current pulse amplitude $I_{p}$ above threshold $I_{t, p}(3,4)$ when the dc currrent $i$ injected in the segment $(1,2)$ is increased. During a short time $t_{w}$, where $t_{w}$ is the pulse width, the total current in segment $(1,2)$ is $\left(I_{p}+i\right)$. For a dc current $i<i_{t}$ and pulse current $I>I_{t, p}$ of the same sense, a weak increase of the overshoot amplitude is observed, then, for $i>i_{t}$, the amplitude of the overshoot decreases and a mixed sluggish and overshoot regime is found. At large values of the dc current $i \gg i_{t}$, the sluggish response dominates. The pulse threshold field $E_{t, p}(3,4)$ decreases as the dc current increases and shows a rather abrupt drop for $i=i_{t}$, as shown in Figure 6 .

When the dc and pulse currents have opposite sense, the change in the overshoot amplitude is barely visible; however, the relaxation at the trailing edge of the pulse is marked by a mixed undershoot and tail regime at large dc current values, as illustrated in Figure 5(b). The effect of the dc current on the transient voltage response between probes $(4,5)$ and $(5,6)$ becomes vanishingly small.

We have investigated the length dependence of the relaxation time $\tau_{\mathrm{on}}$, associated to the overshoot and of

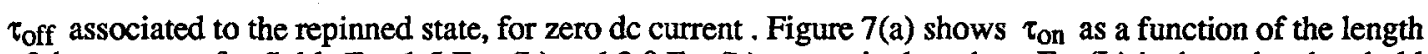
of the segment for fields $E=1.5 \mathrm{E}_{\mathrm{t}, \mathrm{p}}(\mathrm{L})$ and $2.0 \mathrm{E}_{\mathrm{t}, \mathrm{p}}(\mathrm{L})$ respectively, where $\mathrm{E}_{\mathrm{t}, \mathrm{p}}(\mathrm{L})$ is the pulse threshold field of a segment with length $L . \tau_{\mathrm{on}}$ is found to increase with the segment length and obeys an empiric parabolic law. A similar behavior has been reported by Gill (21) in pulse sign memory effects measurements. This indicates a diffusive process for the propagation of $\mathrm{CDW}$ defects with a diffusion constant $D=L^{2} / \tau_{\text {on }} . \tau_{\text {off }}$ is found to decrease monotoneously as a function of $L$, as shown in Figure $7(b)$.

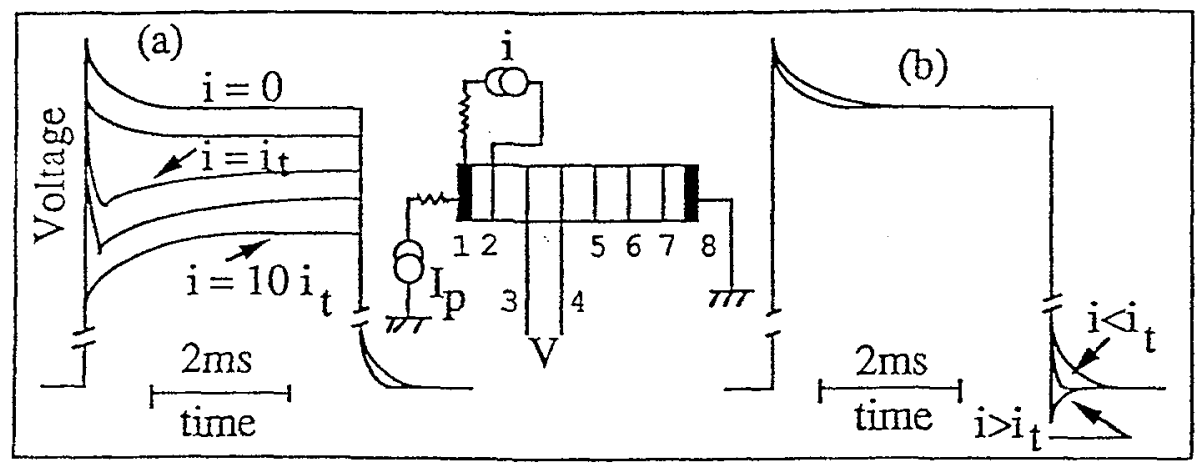

Figure 5. (a) Schematic illustration of the voltage response waveform between probes $(3,4)$ above threshold $E_{t p}(3,4)$ for increasing values of the dc current $i(1,2) ; i(1,2)$ and current pulse $I_{p}$ have the same sense. $i_{t}(1,2)=0.30 \mathrm{~mA}$; threshold pulse current amplitude $I_{t, p}(3,4)=0.36 \mathrm{~mA}$, applied pulse current $I_{p}=$ $2 \mathrm{I}_{\mathrm{t}, \mathrm{p}}$. (b) same as in (a) with dc and pulse current of opposite sense $\mathrm{T}=77 \mathrm{~K}$.

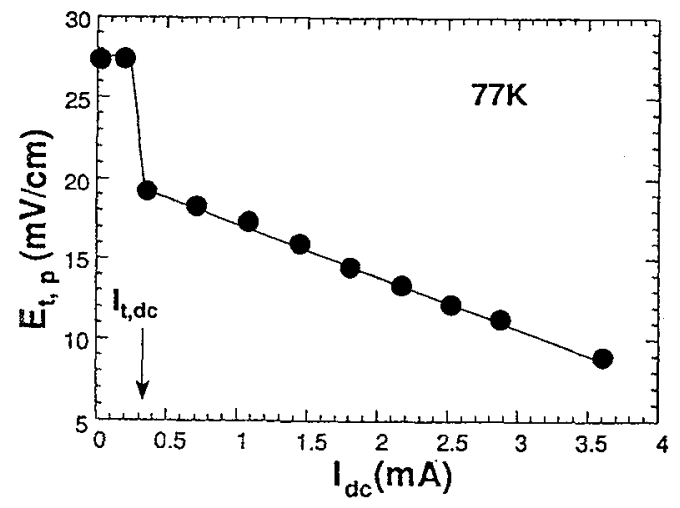
Figure 6. Threshold field Et,p $(3,4)$ as a function of the dc current amplitude injected in segment $(1,2)$. $T=$
$77 \mathrm{~K}$. 

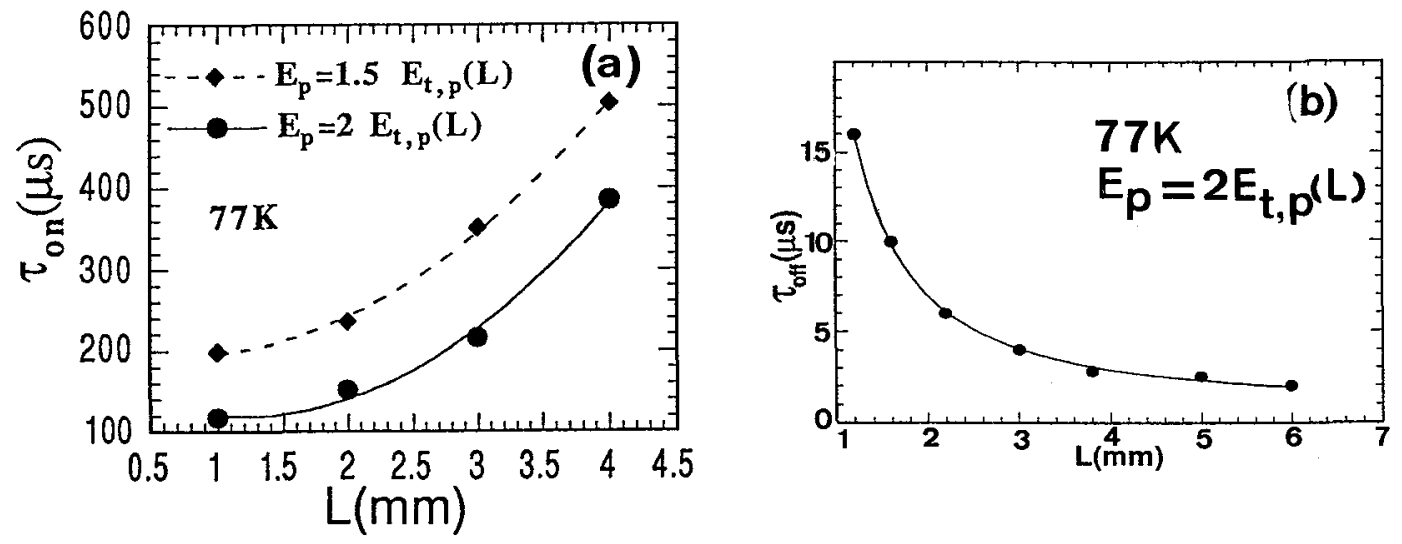

Figure 7. (a) Relaxation time $\tau_{\text {on }}$ of the overshoot as a function of the segment length $L$ for $E p=2 E t, p(\bullet)$ and $\mathrm{Ep}=1.5 \mathrm{Et}, \mathrm{p}(\diamond)$.(b) same as in (a) for relaxation time $\tau_{\text {off }}$ at the trailing edge of the pulse. $\mathrm{T}=77 \mathrm{~K}$.

\section{Discussion}

The results of our study of CDW depinning in pure, non-stoichiometric and V-doped blue bronzes represent additional manifestations of the relaxation of internal CDW deformations due to a sudden change of the applied current. Our results show strong similarities with those found in the time dependent plastic deformation of metals submitted to cyclic loading which result from combined elastic plastic creep motion of dislocations interacting with impurities (22-23). We discuss our results in the context of CDW defects in analogy with strain transient and plastic yielding.In this picture, the depinning is viewed as a shear process and plastic yielding of the CDW, considered as an elastic uniaxial crystal, involves nucleation of CDW dislocation loops (6). Not only preexisting CDW defects might be involved but new CDW defects can be nucleated above threshold (24).

The exact nature of CDW defects involved in these various transient responses is not yet clear. In the plastic yielding picture, the depinning may involve both edge and screw CDW phase dislocations. These two kinds of CDW defects may have different viscosities, since an impurity interacts more easily with an edge dislocation (23). In this context, we propose that the sluggishg behavior would be mainly associated to the motion of CDW edge dislocations, while the overshoot response would correspond mainly to the motion of screw dislocations. In the depinning process, their individual concentration may vary; this would allow for the distinct features identified in the voltage response.

In $\mathrm{V}$-doped samples, the sluggish response $\Delta \mathrm{V}_{\mathrm{S}}$ is more pronounced than in $\mathrm{Rb}_{0.28} \mathrm{MoO}_{3}$; the $\mathrm{CDW}$ transverse coherence length is smaller than in pure samples, due to the induced substitutional disorder (14). This would make the shear deformations of the CDW easier. In the context of the conventional theory of plasticity the fraction $\Delta V_{s}$ would be of the form: $\Delta V_{s}=b A /$ where $b$ is the Burgers vector, $A$ the area swept by a CDW dislocation and 1 the length of a dislocation line pinned to impurity sites. In V-doped samples, 1 may be smaller than in pure samples, leading to a large response $\Delta V_{S}$.

The sequence of pulses illustrated in Figure 1a, with an overshoot followed by sluggish response is just the opposite to that predicted within the Fukuyama-Lee-Rice framework (25). In this approach, the first pulse from a virgin state shows a sluggish response which allows long range phase correlations to build up; the second pulse shows a fast response since long range phase correlations are now frozen. We rather propose that CDW defects might be responsible for this process with a varying concentration, depending on the past electrical history of the sample.

The divergence of $\tau_{\text {on }}$ near threshold together with the divergence of the polarization (26) indicate that the depinning can be viewed as a dynamical critical phenomenon, as predicted by several authors (4) .

The fact that $\tau_{\text {on }}$ is smaller than $\tau_{\text {off }}$ at large values of the current pulse amplitude indicates that the CDW reaches more rapidly the depinned state than the repinned one. In $\mathrm{Rb}_{0.28} \mathrm{MoO}_{3}, \tau_{\text {off }}$ is much larger than in pure samples, indicating a more viscous release of CDW strains.

Under inhomogeneous conditions, as described in section $2 \mathrm{~b}$, we have observed a cross-over from a transient regime dominated by an overshoot to a regime dominated by sluggish response. We propose that 
the influence of a dc current $i(1,2)>i_{t}$ on the transient response waveform in the region $(3,4)$ where the $C D W$ is depinned during a short time $t_{w}$, is due to the growth and motion of phase dislocations loops between the two segments $(1,2)$ and $(3,4)$. The mixed sluggish and overshoot regime at large dc current i> $i_{t}$ may correspond to large strain gradients induced in the moving part of the CDW.

The length dependence of the relaxation times $\tau_{\text {on }}$ and $\tau_{\text {off }}$ may result from non-uniform deformations of the $\mathrm{CDW}$ along the sample, as ascertained from $x$-ray studies of different parts of a sample submitted to current pulses (27).

\section{Conclusion}

In summary, we have investigated the transient CDW dynamics in pure, non-stoichiometric and V-doped blue bronzes. Three kinds of behavior have been found in pure blue bronzes depending on the samples, a weak sluggish response in $\mathrm{Rb}_{0.28} \mathrm{MoO}_{3}$ and a prononced sluggish response in V-doped samples. A critical behavior near threshold for depinning has been observed in pure samples. Induced disorder, either due to V-doping or to a dc electric field applied in a segment adjacent to a segment where the CDW is depinned by pulse currents, leads to a sluggish response. The depinning has been described in terms of plastic flow of CDW edge and screw dislocations which react differently to an applied field.

\section{Acknowledgements}

It is a pleasure to thank J. Marcus and P. Strobel for providing us with blue bronzes samples, R. Buder for his technical help, C. Schlenker and R. Thorne for useful discussions. This work was supported in part by an ECC Science Contract SC1-0032C-CD.

\section{References}

[1] For a review, see " Charge Density Waves in Solids", Modern Problems in Condensed Matter Sciences, vol. 25, eds. L.P. Gor'kov, G. Gruner, (North-Holland, 1989).

[2] Fukuyama H., Lee P.A., Phys. Rev.B 17 (1978) 535.

[3] Lee P.A., Rice T.M., Phys. Rev.B 19 (1985)3970 .

[4] Fischer D.S., Phys. Rev. B 31 (1985) 1396; Sibani P., Littlewood P.B., Phys. Rev. Lett. 64 (1990) 1305.

[5] Ong N.P., Maki K. Phys. Rev. B32, (1985) 6582.

[6] Dumas J., Feinberg D., Europhys. Lett. 2, (1986) 555 .

[7] Mihaly G., Beauchêne P., Marcus J., Dumas J., Schlenker C., Phys. Rev. B 37, (1988) 1047 .

[8] Dumas J., Arbaoui A., Daudin B., Dubus M., Lopes E.B., Almeida M., Synth. Metals 43 (1991) 3813.

[9] Fleming R.M., Schneemeyer L.F., Phys. Rev. B 28 (1983) 6996.

[10] Fleming R.M. Schneemeyer L.F., Phys. Rev. B 33 (1986) 2930 .

[11] Wang Z.Z., Ong N.P., Phys. Rev. B 35 (1987) 5896.

[12] Tsutsumi K., Tamegai T., Kagoshima S., Sato M., J. Phys. Soc. Jpn, 54 (1985) 3004.

[13] Arbaoui A., Dumas J., Lopes E.B., Almeida M., Solid State Commun. 81 (1992) 567 ; Dumas J., Arbaoui A., Ayadi A., Synth. Metals 56 (1993) 2676; Dumas J., Schlenker C., Int. J. Modern Phys.B, to be published (1993).

[14] Girault S., Moudden A.H., Pouget J.P., Godard J.M., Phys. Rev. B 38 (1988) 7980.

[15] Strobel P., Greenblatt M., J. Solid State Chem. 36 (1981) 331.

[16] Zaitsev-Zotov S.V., Synth. Metals 29, F433 (1989); Thorne R., Mc Carren J., DiCarlo D.A., Aldeman T.L., Maher M.P., Synth. Metals 41-43, 3935 (1991).

[17] Gill J.C., Europhys. Lett. 11, 175 (1990).

[18] Saint-Lager M.C., Monceau P., Renard M., Europhys. Lett. 9, 585 (1989).

[19] Janossy A., Mihaly G., Pekker S., Roth S., Solid State Commun. 61, 33 (1987).

120] Csiba T., Kriza G., Janossy A., Phys. Rev. B 40, 10088 (1989).

[21] Gill J.C., Lecture Notes in Physics, vol. 217, (1985), ed. Springer-Verlag, p. 377.

[22] Gibeling J.C., Acta Metall. 37 (1989) 3183.

[23] Mills M.J., Gibeling J.C., Nix W.D., Acta Metall. 33 (1985) 1503.

[24] Bjelis A., Physica Scripta T29 (1989) 62.

[25] Littlewood P.B., Phys. Rev. B 33 (1986) 6694.

[26] Wang Z.Z., Ong N.P., Phys. Rev. Lett. 58 (1987)2375 .

[27] Tsutsumi K., Tamegai T., Kagoshima S., Sato M., in Lecture Notes in Physics, vol, 217, (1985), ed. Springer-Verlag, p. 17. 